\title{
O COMPORTAMENTO ALIMENTAR AOS 30 DIAS DE VIDA ESTÁ ASSOCIADO À ADEQUAÇÃO DO PESO AO NASCIMENTO?
}

\author{
IS EATING BEHAVIOR AT 30 DAYS OF AGE ASSOCIATED \\ WITH ADEQUATE BIRTH WEIGHT?
}

Clin Biomed Res. 2019;39(2):152-160

1 Programa de Pós-graduação em Saúde da Criança e do Adolescente, Faculdade de Medicina, Universidade Federal do Rio Grande do Sul (UFRGS). Porto Alegre, RS, Brasil.

2 Department of Psychiatry, Faculty of Medicine, McGill University. Quèbec, Canada.

3 Faculdade de Medicina, Universidade Federal do Rio Grande do Sul (UFRGS). Porto Alegre, RS, Brasil.

4 Departamento de Pediatria, Faculdade de Medicina, Universidade Federal do Rio Grande do Sul (UFRGS). Porto Alegre, RS, Brasil.

Autor correspondente: Samira Da Cás samiradacas@gmail.com Rua General Rondon, 1344/703. 91900-121, Porto Alegre, RS, Brasil.

\section{Samira Da Cás ${ }^{1}$, Patrícia Pelufo Silveira ${ }^{2}$, Mariana Lopes de Castro ${ }^{3}$, Luciana Friedrich ${ }^{4}$, Elza Daniel de Mello ${ }^{1,4}$}

\section{RESUMO}

Introdução: O objetivo principal é avaliar o comportamento alimentar de recémnascidos (RN) pequenos (PIG) e grandes (GIG) para a idade gestacional através de questionário específico e comparar com $\mathrm{RN}$ adequados para a idade gestacional (AIG) com 1 mês de vida.

Métodos: É um estudo de coorte, cuja primeira fase consistiu na realização de uma entrevista com mães que tiveram seus filhos a termo no Hospital de Clínicas de Porto Alegre. Dados perinatais foram coletados de prontuários eletrônicos. Na segunda fase do estudo, após 1 mês do nascimento, foi aplicado o Questionário sobre Comportamento Alimentar do Bebê (Baby Eating Behaviour Questionnaire, BEBQ) através de contato telefônico.

Resultados: Foram avaliados 126 RN (43 AIG, 43 PIG e 41 GIG). Foi observada uma maior escolaridade em mães de RN PIG $(p=0,004)$ e uma menor prevalência de aleitamento materno exclusivo até a alta hospitalar em RN GIG $(p=0,002)$. A análise de variância não encontrou diferença significativa entre os grupos em relação aos domínios do BEBQ, mesmo quando corrigidos por sexo do RN.

Conclusão: O estudo demonstrou que alterações do comportamento alimentar ainda não estão presentes com 1 mês de vida, sugerindo que não são inatas, e sim desenvolvidas com o passar do tempo. O estudo está limitado a avaliações de crescimento baseadas em registros de terceiros.

Palavras-chave: Comportamento alimentar; restrição de crescimento intrauterino; obesidade

\section{ABSTRACT}

Introduction: The main purpose of this paper was to evaluate the feeding behavior of infants born small and large for gestational age (SGA and LGA, respectively) using a specific questionnaire and to compare them with infants born adequate for gestational age (AGA) at 1 month of age.

Methods: The first phase of this cohort study consisted of an interview with mothers whose babies were born at term at Hospital de Clínicas de Porto Alegre. Perinatal data were collected from electronic medical records. In the second phase of the study, at 1 month of birth, the Baby Eating Behaviour Questionnaire (BEBQ) was administered through telephone interview.

Results: A total of 126 infants (43 AGA, 43 SGA and 41 LGA) with a mean gestational age of 39.4 weeks were assessed. A higher level of education was observed in mothers of SGA infants $(p=0.004)$ and a lower prevalence of exclusive breastfeeding in the 
LGA group ( $p=0.002)$. The analysis of variance found no significant difference between the groups in any of the BEBQ domains, even when corrected for sex of the baby.

Conclusions: This study demonstrated that changes in feeding behavior are not yet present at 1 month of age, suggesting that they are not innate, but rather developed over time. The study is limited to growth assessments based on third-party records.

Keywords: Eating behavior; intrauterine growth restriction; obesity

O peso ao nascer é um forte marcador para aumento da mortalidade e morbidade pós-natal ${ }^{1}$. A velocidade de ganho de peso no início da vida está relacionada ao aumento do índice de massa corporal (IMC) na vida adulta; logo, torna-se fundamental a tomada de medidas preventivas para essa faixa etária ${ }^{2}$. A restrição de crescimento intrauterino (RCIU) é considerada uma disfunção no crescimento fetal, na qual ocorre menor crescimento do que o esperado para aquela fase específica da gestação $0^{3,4}$.

Os RN pequenos (PIG) e grandes (GIG) para a idade gestacional (IG) apresentam fatores de risco aumentados em comparação àquelas crianças que nasceram com peso adequado para a idade gestacional (AIG), como por exemplo a regulação prejudicada da glicose ${ }^{5}$, o aumento da pressão arterial sistêmica ${ }^{6}$, a diminuição da função pulmonar $^{7}$ e a menor densidade óssea ${ }^{8}$. Tais agravos podem levar a doenças crônicas como obesidade, hipertensão arterial sistêmica, osteoporose, diabetes tipo II, doenças cardiovasculares, e acidente vascular cerebral ${ }^{9}$.

Segundo a teoria de Barker, também conhecida como "teoria da origem fetal das doenças do adulto", a deficiência nutricional na gravidez e no início da vida pode levar a adaptações metabólicas e estruturais permanentes ${ }^{10}$. Estudos recentes demonstram que insultos vivenciados no início da vida, como a restrição de crescimento intrauterino (RCIU), são capazes de programar o comportamento alimentar na vida adulta. Esses indivíduos têm maior preferência por alimentos palatáveis, que são alimentos ricos em açúcar e/ ou gorduras, em comparação a frutas e verduras/legumes ${ }^{11-14}$.

$\mathrm{Na}$ tentativa de compreender o comportamento alimentar de crianças foi desenvolvido um instrumento de avaliação para lactentes até 6 meses, chamado Questionário sobre o Comportamento Alimentar de Bebês ("Baby Eating Behaviour Questionnaire", $B E B Q$ ), que se propõe a avaliar 4 traços de apetite que parecem influenciar o ganho de peso: enjoyment of food (prazer em comer), food responsiveness (resposta à comida), slowness in eating (ingestão lenta), e satiety responsiveness (resposta à saciedade) ${ }^{15,16}$. As variáveis do questionário que indicam um maior apetite ou um maior interesse pelo alimento são medidas pelos seguintes domínios: resposta à comida, que indica a resposta da criança em querer comer; prazer em comer, que capta o nível de prazer subjetivo ao alimentar-se. As variáveis que avaliam traços relacionados ao melhor controle do apetite e ao menor interesse no alimento são: ingestão lenta, que avalia o tempo que a criança leva para se alimentar, e resposta à saciedade, que mede a plenitude de satisfação da criança, mostrando quando ela já está com o estômago cheio ${ }^{17}$.

A validação deste instrumento para a língua portuguesa foi realizada anteriormente a este estudo ${ }^{18}$ e a análise psicométrica foi realizada simultaneamente ao presente estudo (Basquerote et al. ahead of print), conferindo suporte para o uso das escalas do Baby Eating Behaviour Questionnare.

Este estudo teve como objetivo avaliar o comportamento alimentar de lactentes nascidos PIG e GIG, e compará-lo aos RN AIG. A hipótese é de que as crianças nascidas pequenas e grandes para a idade gestacional podem apresentar um padrão de comportamento alimentar em idade tão precoce quanto 1 mês de vida ${ }^{12,14}$.

\section{MÉTODOS}

Estudo de coorte, que incluiu RN internados junto a suas mães no alojamento conjunto do Hospital de Clinicas de Porto Alegre (HCPA), entre o período de março de 2016 a março de 2017. Nascem em média 300 bebês por mês no Alojamento Conjunto.

Foram excluídos os RN que apresentavam malformações congênitas craniofaciais ou outras malformações maiores que interferissem com a alimentação, síndromes genéticas, além dos neonatos expostos à infecção por HIV e outras infecções congênitas neonatais do grupo STORCH (sífilis, toxoplasmose, hepatites, rubéola, citomegalovírus e herpes) suspeitas ou confirmadas. Os pré-termos também foram excluídos, além daqueles $\mathrm{RN}$ que estiveram internados em UTI neonatal por qualquer período ou motivo. Nenhum RN foi excluído devido a patologias maternas específicas.

Todos os dias eram realizadas revisões da lista dos nascidos vivos que não necessitavam de internação e esses eram divididos entre os grupos PIG, GIG e AIG para recrutamento. Os RN que entraram no estudo foram divididos em 3 grupos:

- Grupo PIG: aqueles com peso de nascimento abaixo do percentil 10 de acordo com a curva de 
Alexander ${ }^{4}$, que é a padronizada no Hospital de Clínicas de Porto Alegre, de acordo com sexo e IG;

- Grupo GIG: constituído por aqueles que nasceram com peso acima do percentil 90 da mesma curva de Alexander;

- Grupo Controle: RN nascidos AIG e que nasceram no mesmo período do estudo.

A IG selecionada foi a ecografia gestacional precoce (antes de 12 semanas de gestação) ou o exame físico do RN (Método de Capurro ${ }^{19}$ ), caso não houvesse ecografia ou caso a mesma tenha sido realizada após as 12 semanas de gestação.

$O$ estudo consistiu em duas fases:

- Primeira fase: O primeiro contato com as mães foi realizado no alojamento conjunto do HCPA em até 24 horas após o parto. As mães dos RN selecionados foram convidadas a participar do estudo e as que aceitaram, assinaram o Termo de Consentimento Livre e Esclarecido (TCLE). Neste momento foi aplicado um questionário para obtenção de informações socioeconômicas e de escolaridade. Os dados referentes à gestação e ao nascimento foram coletados do prontuário eletrônico;

- Segunda fase: Quando o RN estivesse com 30 dias e vida era realizado contato telefônico para a aplicação do questionário BEBQ. Além disso, foram também coletados dados acerca do peso e comprimento atuais do lactente e a data desta última pesagem (dados que constam na Caderneta de Saúde).

As variáveis referentes às mães e seus $\mathrm{RN}$ foram:

- Sexo, idade materna. Tabagismo materno (definido como uso de qualquer quantidade de cigarros em qualquer período da gestação), nível sócio-econômico (definido de acordo com a tabela da ABEP - Associação Brasileira de Empresas de Pesquisa ${ }^{20}$, classificado em níveis $A$ a $E$ ), escolaridade materna, via de parto, IG (semanas), Escore-z do peso ao nascimento, Escore de Apgar no $1^{\circ}$ e no 5 - minutos, percentual de amamentação na $1^{\text {a }}$ hora de vida, de amamentação exclusiva em Alojamento Conjunto e no $1^{\circ}$ mês de vida.

IMC foi definido como peso $(\mathrm{kg}) / \mathrm{comprimento}(\mathrm{cm})^{2}$.

\section{Análise Estatística}

Considerando-se médias e desvios-padrão de variáveis do comportamento alimentar avaliadas em crianças pré-escolares nascidas com RCIU ou não ${ }^{21}$ através um questionário correspondente para esta faixa etária, (Children Eating Behavior
Questionnaire, CEBQ, Wardle) $)^{22}$, foi calculado um tamanho amostral por grupo e por sexo usando o site da University of British Columbia, Canada ${ }^{23}$. Para um erro alfa de 0.05 e um poder de $95 \%$, estimou-se que seriam necessárias 20 crianças por grupo para captar diferenças entre os grupos. Esse número de crianças foi dobrado durante a coleta de dados, para garantir que uma possível diferença entre os grupos fosse realmente encontrada.

Os dados paramétricos foram expressos através de média \pm desvio padrão. Para análise das variáveis antropométricas, foram utilizadas as medidas em z-escore de acordo com os padrões de crescimento da Organização Mundial de Saúde ${ }^{24}$, de forma que meninos e meninas pudessem ser analisados em conjunto. Além disso, escores-z de índice de massa corporal (IMC) ao nascimento ajustadas para a IG foram calculadas de acordo com o International Fetal and Newborn Growth Consortium for the 21st Century25. A diferença (delta) entre o escore-z com 1 mês e ao nascimento foi usada como uma medida de crescimento. O teste de Kolmogorov-Smirnov foi usado para avaliar a distribuição das variáveis do $B E B Q$, e quando significativo, estas foram log-transformadas para análise.

Foi realizada uma análise comparando o grupo classificado como PIG pela curva de Alexander, com outras curvas utilizadas correntemente para esta classificação (Pedreira, Intergrowth, WHO, Victora e Kramer), para garantirmos que os resultados do estudo não fossem minimizados devido à curva escolhida.

As comparações entre os grupos do estudo foram realizadas através de análise de variância (ANOVA), seguida do teste de comparações múltiplas de Student-Newman-Keuls (SNK), quando indicado. As ANOVAs foram ajustadas para a idade do RN na data de sua pesagem mais próxima de 30 dias, e pelo tipo de alimentação (leite materno exclusivo, leite materno complementado com fórmula láctea ou leite de vaca, ou fórmula láctea ou leite de vaca).

Para análise dos dados de forma contínua, utilizamos a razão de crescimento fetal $(\mathrm{RCF}=$ peso ao nascer $/ \mathrm{média}$ populacional do peso ao nascer específica para sexo e IG) utilizando a curva nacional de Pedreira ${ }^{26}$. As correlações entre a RCF e os dados de crescimento e de comportamento alimentar foram realizadas através do Teste de Pearson ou regressão linear com análise de resíduos quando indicado. Foram considerados estatisticamente significativos resultados com $\mathrm{p}<0,05$.

Aclassificação sócio-econômica foi estratificada de acordo com a tabela da ABEP, que classifica as classes entre $A$ (classe mais alta) e $E$ (classe mais baixa), de acordo com a quantidade de veículos e eletrodomésticos presentes no domicílio, encanamento de água, asfalto na rua e escolaridade do chefe da família. 


\section{Considerações Éticas}

A pesquisa foi submetida e aprovada pelo Comitê de Ética em Pesquisa com Seres Humanos do Hospital de Clínicas de Porto Alegre sob o n 16-0106. Todos os participantes do estudo assinaram o TCLE.

\section{RESULTADOS}

A amostra do estudo foi inicialmente constituída por $127 \mathrm{RN}$, distribuídos nos 3 grupos de recrutamento: 43 PIG, 43 AIG e 41 GIG. As características descritivas da amostra são demonstradas na Tabela 1. Na segunda fase do estudo (entrevista telefônica) ocorreram perdas por insucesso no contato com as mães, ocorrendo estas perdas no percentual de $16,3 \%$ do grupo PIG e $11,6 \%$ do grupo AIG. Não houve nenhuma perda no grupo GIG.

O recrutamento deste projeto foi realizado visando a inclusão de RN pertencentes aos três grupos de crescimento fetal: PIG < percentil 10, percentil $10<\mathrm{AIG}>$ percentil 90 e GIG > percentil 90 da curva de Alexander ${ }^{4}$. A Tabela 2 demonstra o percentual de concordância em relação à classificação AIG/GIG/PIG entre a curva de Alexander e outras correntemente utilizadas (Tabela 2).

Analisando a relação entre os três grupos de crescimento fetal estabelecidos de acordo com o recrutamento (AIG, PIG e GIG conforme a curva de Alexander ${ }^{4}$ ) e o sexo nos desfechos de comportamento alimentar avaliados pelo BEBQ, não foram observadas diferenças entre os grupos,

Tabela 1: Características da amostra.

\begin{tabular}{|c|c|c|c|c|}
\hline Grupo & $\begin{array}{c}\text { AIG } \\
(n=43)\end{array}$ & $\begin{array}{c}\text { PIG } \\
(n=43)\end{array}$ & $\begin{array}{c}\text { GIG } \\
(n=41)\end{array}$ & $\mathbf{P}$ \\
\hline Sexo feminino & $53,5 \%$ & $58,1 \%$ & $34,1 \%$ & $0,081^{\mathrm{b}}$ \\
\hline Idade materna (anos) & 24,9 & 26,1 & 28,2 & $0,086^{b}$ \\
\hline Tabagismo materno & $11,6 \%$ & $20,9 \%$ & $17,1 \%$ & $0,495^{b}$ \\
\hline Nível sócio-econômico A-Bc & $20,9 \%$ & $27,9 \%$ & $19,5 \%$ & $0,888^{b}$ \\
\hline Escolaridade materna $<9$ anos & $53,7 \%$ & $37,2 \%$ & $46,2 \%$ & $0,490^{\mathrm{b}}$ \\
\hline Nascimento por cesárea & $16,3 \%$ & $27,9 \%$ & $68,3 \%$ & $<0,0001^{b}$ \\
\hline Idade gestacional (semanas) & $39,3 \pm 0,9$ & $39,3 \pm 1,0$ & $39,8 \pm 1,1$ & $0,052^{\mathrm{a}}$ \\
\hline Escore-z peso ao nascimento & $0,12 \pm 0,62$ & $-1,40 \pm 0,41$ & $0,43 \pm 3,00$ & $<0,0001^{a}$ \\
\hline Média peso ao nascimento & $3351,2 \pm 294,7$ & $2677,3 \pm 160,3$ & $4204,5 \pm 254,6$ & $<0,01^{\mathrm{a}}$ \\
\hline APGAR $1^{\circ}$ minuto & $8,4 \pm 1,3$ & $8,3 \pm 1,3$ & $8,2 \pm 1,3$ & $0,859^{a}$ \\
\hline APGAR $5^{\circ}$ minuto & $9,3 \pm 0,9$ & $9,4 \pm 0,7$ & $9,2 \pm 0,7$ & $0,479^{a}$ \\
\hline Amamentação $1^{\circ}$ hora vida & $61,9 \%$ & $69,8 \%$ & $56,1 \%$ & $0,592^{\mathrm{b}}$ \\
\hline AME no AC & $85,7 \%$ & $62,8 \%$ & $48,8 \%$ & $<0,0001^{b}$ \\
\hline AME $1^{\circ}$ mês de vida & $88,4 \%$ & $69,8 \%$ & $29,9 \%$ & $0,056^{\mathrm{a}}$ \\
\hline
\end{tabular}

Tabela 2: Classificação de peso para a idade gestacional segundo outras referências.

\begin{tabular}{|c|c|c|c|c|}
\hline Critério & Referência & PIG \% & AIG \% & GIG \% \\
\hline $\begin{array}{l}\text { Peso ajustado para idade gestacional } \\
\text { PIG<p10, GIG>p90 }\end{array}$ & (Alexander et al., 1996) & 100 & 100 & 100 \\
\hline $\begin{array}{l}\text { Peso ajustado para idade gestacional } \\
P I G<p 10, G I G>p 90\end{array}$ & (Pedreira et al., 2011) & 44,2 & 95,3 & 90 \\
\hline $\begin{array}{l}\text { Peso ajustado para idade gestacional } \\
\text { PIG<p10, GIG>p90 }\end{array}$ & $\begin{array}{l}\text { (Villar et al., 2014) } \\
\text { Intergrowth 21th }\end{array}$ & 48,8 & 88,4 & 92,5 \\
\hline $\begin{array}{l}\text { Razão de crescimento fetal } \\
P I G<0,85, G \mid G>1,15\end{array}$ & $\begin{array}{l}\text { (Kramer; Platt et al. apud } \\
\text { Pedreira et al., 2011) }\end{array}$ & 72,1 & 90,7 & 97,5 \\
\hline $\begin{array}{l}\text { Razão de crescimento fetal } \\
P I G<0,85, G \mid G>1,15\end{array}$ & $\begin{array}{l}\text { (Kramer; Platt et al. apud } \\
\text { Villar et al., 2014) } \\
\text { Intergrowth 21th }\end{array}$ & 62,8 & 86 & 92,5 \\
\hline $\begin{array}{l}\text { Escore-z IMC ajustado para idade gestacional } \\
(\text { WHO, 2006 })^{24} \\
\text { PIG<-1, GIG>+1 }\end{array}$ & (Villar et al., 2014) $)^{25}$ & 44,2 & 81,4 & 82,9 \\
\hline $\begin{array}{l}\text { IMC ajustado para idade gestacional } \\
\text { PIG<p3, GIG>p97 }\end{array}$ & $\begin{array}{l}\text { (Victora; Villar et al. apud } \\
\text { Villar et al., 2014) }\end{array}$ & 4,7 & 100 & 57,5 \\
\hline
\end{tabular}


efeito do sexo ou interações. AANOVA de duas vias (grupo de crescimento fetal e sexo como fatores), ajustada para tipo de alimentação, idade da criança e escore-z de IMC com 1 mês de vida não demonstrou resultados estatisticamente significativos.

Avaliando-se o crescimento fetal de forma contínua através da RCF (e não em grupos AIG/PIG/GIG), também não se observou correlação entre o crescimento fetal e os escores de comportamento alimentar avaliados pelo BEBQ.

Ao avaliar o delta de ganho ponderal entre o nascimento e o primeiro mês de vida (delta escore $Z$ de IMC entre 1 mês e ao nascimento), observa-se uma correlação negativa entre a RCF e o delta escore-z de IMC (Correlação de Pearson, $r=-0,419$, $\mathrm{P}<0.0001)$, demonstrando-se que quanto menor $\mathrm{o}$

Tabela 3: Correlações entre o ganho ponderal no primeiro mês de vida e os domínios do comportamento alimentar avaliados pelo Baby Eating Behaviour Questionnaire.

\begin{tabular}{lcc}
\hline \multicolumn{1}{c}{ Domínios BEBQ } & $\begin{array}{c}\text { Delta-z de } \\
\text { IMC (r) }\end{array}$ & P \\
\hline Prazer em comer & $-0,083$ & 0,393 \\
Resposta à comida & $-0,133$ & 0,167 \\
Ingestão lenta & $-0,095$ & 0,328 \\
Resposta à saciedade & 0,040 & 0,682 \\
\hline
\end{tabular}

Legenda: $\mathrm{BEBQ}=$ Baby Eating Behaviour Questionnaire; $\mathrm{IMC}=$ índice de massa corporal; $\mathrm{P}=$ probabilidade de significância. crescimento fetal, maior o crescimento no primeiro mês de vida. No entanto, não há correlação entre o ganho ponderal e os escores de comportamento alimentar avaliados pelo BEBQ (Tabela 3). Ainda que não se tenha conseguido demonstrar diferença entre os padrões de comportamento alimentar e os grupos PIG, GIG e AIG, observou-se um resultado significativo inverso entre o crescimento fetal e a velocidade de ganho de peso no primeiro mês de vida.

Ao categorizar os RN de acordo com o sinal do delta escore- $z$ de IMC (negativo, para lactentes que diminuíram de escore-z, e positivo, para os que aumentaram o escore-z entre o nascimento e 1 mês de vida), similarmente não se observam diferenças entre os grupos com relação aos desfechos de comportamento alimentar. O teste ANOVA de duas vias (grupo de IMC e sexo como fatores), mesmo ajustado para tipo de alimentação, idade da criança e RCF mostra que não há efeitos do grupo de IMC, nem do sexo, nem interação grupo vs. sexo nos desfechos Prazer em Comer, Resposta à Saciedade e Ingestão Lenta do BEBQ. No entanto, há uma interação entre grupo e sexo no domínio Resposta à comida, na qual meninos que apresentam aumento no IMC tem maior Resposta à comida do que meninos que apresentam diminuição do IMC, enquanto que nas meninas o efeito é oposto (interação sexo e grupo, ANOVA de duas vias, $F(1,101)=3,897, p=0,05)$ (Figura 1).
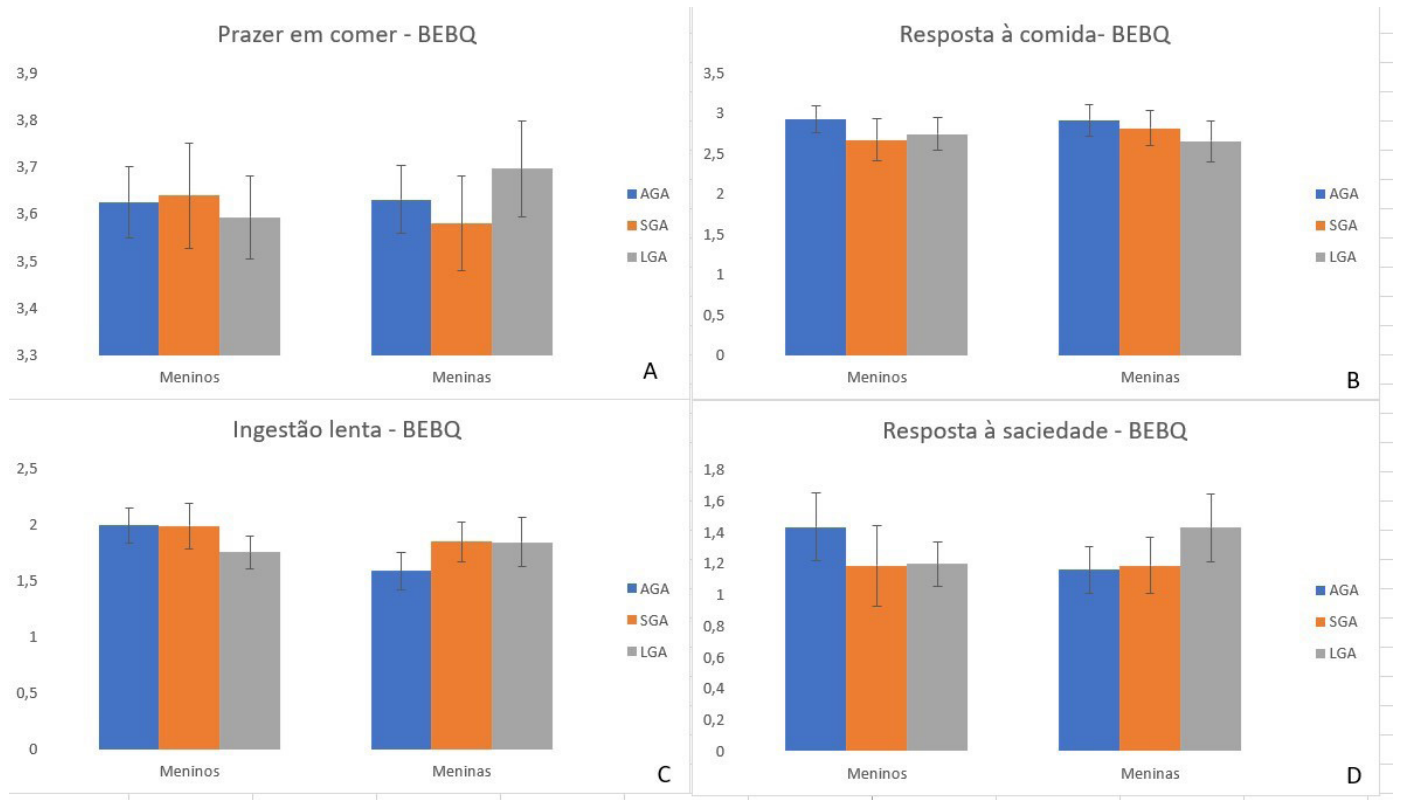

Figura 1: Relação entre a inclinação do ganho ponderal em escores-z no primeiro mês de vida e o sexo nos desfechos do BEBQ. (A) Prazer em comer, ANOVA de duas vias: grupo $[F(1,107)=0,70, p=0.792]$, sexo $[F(1,107)=0,023$, $p=0.879]$ e interação $[F(1,107)=2,018, p=0.159]$; $(B)$ Resposta à comida, grupo $[F(1,107)=0,346, p=0,558]$ e sexo $[F(1,107)<0,0001, p=0,986]$, interação $[F(1,107)=3,897, p=0,05] ;(C)$ Ingestão lenta: grupo $[F(1,106)=1,525$, $p=0,220]$, sexo $[F(1,106)=1,934, p=0,167]$ e interação $[F(1,106)=0,408, p=0,525]$ e $(D)$ Resposta à saciedade: grupo $[F(1,107)=0,185, p=0,668]$, sexo $[F(1,107)=0,165, p=0,665]$ e interação $[F(1,107)=1,679$, $p=0,198]$. Dados são expressos como média \pm erro padrão. Legenda: BEBQ = Baby Eating Behavior Questionnaire. 


\section{DISCUSSÃO}

A análise da amostra do presente estudo encontrou um escore $Z$ de peso de nascimento significativamente diferente entre os grupos em estudo, o que era esperado. A diferença estatisticamente significativa de um maior número de partos cesarianas nos RN GIGs também era esperada. Os demais dados demográficos não apresentaram diferenças significativas entre os grupos em estudo. No entanto, a amamentação exclusiva dentro do hospital foi significativamente menor nos RN GIG do que nos demais, possivelmente pelo fato de que estes RN nascem mais frequentemente por parto cesariana, suas mães demoram mais a conseguir amamentar $e$ estes bebês podem ser mais vorazes e ainda, mais suscetíveis à hipoglicemia neonatal, o que faz com que recebam mais fórmula láctea enquanto estão internados.

A média de internação na Unidade de Alojamento Conjunto foi, para todos os grupos, entre 48 e 72 horas. Um dos resultados do presente trabalho foi a existência de diferenças no AME entre os grupos estudados, ainda no alojamento conjunto. Na primeira hora de vida o grupo que mais mamou ao seio materno foi o grupo PIG, seguido do grupo controle (AIG) e por último o grupo GIG. Contudo, durante a permanência no alojamento conjunto houve um aumento de AME nos AIG em relação aos PIG. O grupo GIG permaneceu recebendo menos AME. Ao realizarmos o seguimento no primeiro mês de vida, observamos que quase todas os lactentes AIG permaneceram em AME, seguidos pelo grupo de PIG e, com uma diminuição significativa do AME no grupo GIG. Normalmente estes lactentes são percebidos como "vorazes" pelas mães, equipe médica e de enfermagem, além de serem grupo de risco para hipoglicemia neonatal, e acabam por receber precocemente fórmula láctea já na maternidade, prática esta que se mantém após a alta. O estudo de Smith \& Becker analisou dois grupos: um recebendo AME na maternidade e outro grupo que teria recebido água ou solução glicosada nos primeiros dias após nascimento. Além dos autores não perceberem nenhum benefício em relação às medidas de glicemia, temperatura e perda de peso, foi observada uma taxa de AME maior no grupo que foi exclusivamente amamentado na maternidade ${ }^{27}$.

O presente estudo também observou que quanto menor o crescimento fetal e o peso de nascimento, maior é o ganho ponderal no primeiro mês de vida $(\triangle \mathrm{IMC})$. No entanto, não se conseguiu demonstrar a associação entre peso de nascimento, $\Delta \mathrm{IMC}$ e os escores de comportamento alimentar do questionário $B E B Q$, mesmo quando ajustados para sexo e grupos, tipo de alimentação e escore $Z$ de IMC com 1 mês de vida. $O$ questionário foi aplicado com 1 mês de vida, e possivelmente as alterações descritas devam ocorrer após este período inicial, sendo que o presente estudo não foi capaz de detectar estes achados em um momento tão precoce. Comparando com a literatura, vimos que estudo conduzido por Stettler, et al. ${ }^{28}$, também mostra que ser PIG e apresentar uma recuperação muito rápida do ganho ponderal pode levar a um risco aumentado de desenvolver síndrome metabólica na vida adulta. $\mathrm{O}$ alto peso ao nascer também está associado, bem como o rápido e precoce ganho de peso e a baixa duração da amamentação, ao ganho de peso excessivo e à obesidade na infância e na vida adulta ${ }^{28,29}$.

Quando comparada a curva de Alexander ${ }^{4}$, elaborada nos EUA, utilizada como padrão no presente estudo para a classificação dos grupos AIG, PIG e GIG -, utilizada como padrão na assistência no HCPA -, com a curva brasileira de Pedreira ${ }^{30}$ e com a curva de crescimento fetal preconizada pela Organização Mundial de Saúde, a Intergrowth ${ }^{25}$, existe uma concordância considerável entre os classificados como AIG e GIG. No entanto, menos de $50 \%$ daqueles neonatos classificados como PIG pela curva de Alexander continuariam sendo PIG pela classificação de Pedreira ou da Intergrowth. O mesmo ocorre para a razão de crescimento fetal e para o escore $Z$ de IMC, ambos apresentando pouca concordância entre as curvas. Pode-se imaginar que o grupo de PIGs do presente estudo (pela curva de Alexander) pudesse conter pacientes considerados AIG pelas outras curvas e, devido a esse achado, não teria se encontrado diferença no padrão de comportamento alimentar deste grupo. Como a curva de Alexander é utilizada por muito tempo em vários serviços de neonatologia no mundo, os autores não acreditam que esta possa ser a explicação para não se ter encontrado diferença no comportamento alimentar deste grupo PIG quando comparado aos outros grupos.

Trabalhos da literatura mostram que há uma relação entre o crescimento fetal e a preferência alimentar por carboidratos e gordura ao longo da vida. Esta situação é observada em um estudo ${ }^{14}$ que correlaciona o crescimento intrauterino e a resposta hedônica ao doce em RN prematuros no seu primeiro dia de vida. Ainda, nesse trabalho foi observado que os neonatos que apresentaram RCIU têm uma diminuição na sensibilidade ao prazer relacionado ao consumo de alimentos doces, levando a um maior consumo desses. Em outro estudo foram avaliados, aos 24 anos, 2063 participantes de uma coorte preexistente de brasileiros RN, cujo objetivo era investigar se a RCIU estava relacionada a determinadas preferências alimentares. Foi encontrada associação positiva em mulheres que demostraram preferência por carboidratos a proteínas, bem como relação cintura-quadril maior nesse grupo, quando comparadas ao grupo controle, ocorrendo maior risco 
dessas mulheres de desenvolver síndrome metabólica ao longo do tempo ${ }^{13}$. Seguindo na mesma linha, Silveira, et al. investigaram a relação entre peso ao nascer e o impulso em comer em crianças de três anos de idade. $\mathrm{O}$ estudo apontou que meninas que nasceram com o peso normal mostraram melhor habilidade em atrasar o impulso por recompensas alimentares do que meninos. Por outro lado, no grupo de RCIU não houve diferenças entre os sexos, constatando-se, ainda, maior impulsividade por doces e alimentos palatáveis por meninas que apresentavam um IMC maior aos 48 meses de idade em contraste com crianças que nasceram com peso normal ${ }^{31}$. Em um estudo utilizando o questionário $B E B Q$, Quah e colaboradores observaram que uma maior responsividade alimentar aos 3 meses estava associada a um maior IMC e ganho de peso dos 6 meses aos 15 meses de idade. Por outro lado, lentidão ao comer e maior responsividade à saciedade estavam associados a um IMC menor ${ }^{30}$.

No presente estudo empregou-se o BEBQ buscando aferir as diferenças no comportamento alimentar em diferentes grupos de RN, sendo um fator diferencial a sua aplicação em fase precoce do desenvolvimento humano. Embora os estudos citados anteriormente tenham consistentemente demostrado que o crescimento fetal afeta a preferência alimentar, na presente avaliação, onde o questionário foi aplicado com 1 mês de vida, não foram observadas alterações significativas quanto à preferência alimentar neste momento. Entretanto, no que diz respeito ao padrão do comportamento alimentar, no critério Resposta à Comida foi observada uma correlação direta entre este domínio e o aumento no IMC no sexo masculino. Já no sexo feminino, verificou-se o efeito inverso. Nota-se que o crescimento no primeiro mês de vida parece ter relação com o comportamento alimentar de forma interativa com o sexo, desempenhando papel mais importante que o crescimento fetal na determinação do perfil de comportamento alimentar. Dessa forma, se supõe que o crescimento fetal impacte na preferência, enquanto o crescimento pós-natal influencia o padrão de comportamento alimentar.

O estudo tem como limitação a época precoce em que foi aplicado o questionário $B E B Q$, assim como o fato do recrutamento ter sido realizado em um único centro hospitalar e as avaliações do crescimento baseadas em registros de terceiros. O BEBQ é um relato materno e subjetivo, e portanto, pode sofrer influência de flutuações do humor materno, que não foi avaliado neste trabalho.

Sabe-se que não existe, até o momento, uma técnica única capaz de melhor avaliar o comportamento alimentar em lactentes. No entanto, o questionário $B E B Q$ tem sido amplamente utilizado na literatura e já foi validado para a língua portuguesa. A comparação dos valores encontrados no $B E B Q$ com medidas mais objetivas do apetite, como marcadores bioquímicos, pode, no futuro, trazer mais riqueza para os dados encontrados.

Por fim, apesar de este estudo não ter sido capaz de detectar alterações de comportamento alimentar tão precoce quanto 1 mês de idade, conseguimos encontrar alguns resultados consistentes com a literatura, como algumas diferenças entre sexos e a relação inversa entre crescimento fetal/peso ao nascimento e ganho de peso pós-natal. Estes resultados estimulam a seguir investigando 0 comportamento alimentar de lactentes para que se possa entender melhor a questão da obesidade e da síndrome metabólica na infância e na vida adulta.

\section{Agradecimentos}

Nosso profundo agradecimento às famílias que participaram da pesquisa. Declaro que os autores são internamente responsáveis pelo conteúdo cientifico do documento.

Fonte de Financiamento: Nenhum.

Conflitos de Interesse: Os autores declaram não ter conflitos de interesse.

\section{REFERÊNCIAS}

1. Sochet AA, Ayres M, Quezada E, Braley K, Leshko J, Amankwah EK et al. The importance of small for gestational age in the risk assessment of infants with critical congenital heart disease. Cardiol Young. 23(6):896904. http://dx.doi.org/10.1017/ S1047951113001960.

2. Bjerregaard LG, Rasmussen KM, Michaelsen KF, Skytthe A, Mortensen $\mathrm{EL}$, Baker JL, et al. Effects of body size and change in body size from infancy through childhood on body mass index in adulthood. Int $J$ Obes. 2014;38(10):1305-11. http:// dx.doi.org/10.1038/ijo.2014.108. PMid:24942870.

3. Chatelain P. Children born with intrauterine growth retardation (IUGR) or small for gestational age (SGA): long term growth and metabolic consequences. Endocr Regul. 2000;34(1):33-6. PMid:10808251.
4. Alexander GR, Himes JH, Kaufman RB, Mor J, Kogan M. A United States national reference for fetal growth. Obstet Gynecol. 1996;87(2):163-8. http://dx.doi.org/10.1016/00297844(95)00386-X. PMid:8559516.

5. de Bruin CDE, van der Lugt NM, Visser R, Oostdijk W, van Zwet EW, te Pas $A B$, et al. Dysglicaemia in small-forgestational-age neonates: a matched case-control study in monochorionic twins. J Matern Fetal Neonatal Med. 
2016;29(13):2114-9. http://dx.doi.org /10.3109/14767058.2015.1076787. PMid:26365253.

6. Panaitescu AM, Baschat AA, Akolekar $\mathrm{R}$, Syngelaki A, Nicolaides $\mathrm{KH}$. Association of chronic hypertension with birth of small for gestational age neonates. Ultrasound Obstet Gynecol. 2017;50(3):361-6. http://dx.doi. org/10.1002/uog.17553.

7. Nobile S, Marchionni P, Carnielli VP. Neonatal outcome of small for gestational age preterm infants. Eur J Pediatr. 2017;176(8):1083-8. http:// dx.doi.org/10.1007/s00431-017-29571. PMid:28660312.

8. Kuh D, Wills AK, Shah I, Prentice A, Hardy R, Adams JE, et al. Growth from birth to adulthood and bone phenotype in early old age: a british birth cohort study. J Bone Miner Res. 2014;29(1):123-33. http:// dx.doi.org/10.1002/jbmr.2008. PMid:23761289.

9. Portella AK, Silveira PP. Neurobehavioral determinants of nutritional security in fetal growthrestricted individuals. Ann $N$ Y Acad Sci. 2014;1331(1):15-33. http:// dx.doi.org/10.1111/nyas.12390. PMid:24650246.

10. Barker DJ, Eriksson JG, Forsén T, Osmond C. Fetal origins of adult disease: strength of effects and biological basis. Int $\mathrm{J}$ Epidemiol. 2002;31(6):1235-9. http://dx.doi. org/10.1093/ije/31.6.1235. PMid:12540728.

11. Lussana F, Painter RC, Ocke MC, Buller HR, Bossuyt PM, Roseboom TJ. Prenatal exposure to the Dutch famine is associated with a preference for fatty foods and a more atherogenic lipid profile. Am J Clin Nutr. 2008;88(6):1648-52. http:// dx.doi.org/10.3945/ajcn.2008.26140. PMid:19064527.

12. Barbieri MA, Portella AK, Silveira PP, Bettiol H, Agranonik M, Silva $A A$, et al. Severe intrauterine growth restriction is associated with higher spontaneous carbohydrate intake in young women. Pediatr Res. 2009;65(2):215-20. http://dx.doi. org/10.1203/PDR.0b013e31818d6850. PMid:19047956.

13. Ayres C, Agranonik M, Portella AK, Filion F, Johnston CC, Silveira PP. Intrauterine growth restriction and the fetal programming of the hedonic response to sweet taste in newborn infants. Int $\mathrm{J}$ Pediatr. 2012;2012:657379:1-5. http:// dx.doi.org/10.1155/2012/657379. PMid:22851979.

14. Perälä MM, Männistö $S$, Kaartinen NE, Kajantie E, Osmond C, Barker DJ, et al. Body size at birth is associated with food and nutrient intake in adulthood. PLoS One. 2012;7(9):e46139. http://dx.doi. org/10.1371/journal.pone.0046139. PMid:23049962.

15. Sleddens EF, Kremers SP, Thijs C. The children's eating behaviour questionnaire: factorial validity and association with Body Mass Index in Dutch children aged 6-7. Int J Behav Nutr Phys Act. 2008;5(1):49. http:// dx.doi.org/10.1186/1479-5868-5-49. PMid:18937832.

16. Spence JC, Carson V, Casey L, Boule $\mathrm{N}$. Examining behavioural susceptibility to obesity among Canadian pre-school children: the role of eating behaviours. Int J Pediatr OBES. 2011;6(Supl 3), e501-7.

17. Llewellyn $\mathrm{CH}$, Van Jaarsveld $\mathrm{CH}$, Johnson L, Carnell S, Wardle J. Development and factor structure of the Baby Eating Behaviour Questionnaire in the Gemini birth cohort. Appetite. 2011;57(2):388-96. http://dx.doi. org/10.1016/j.appet.2011.05.324. PMid:21672566.

18. Lucion MK, Kliemann N, Bernardi FR, Loreto BBL. Translation and semantic validity of the BABY EATING BEHAVIOUR QUESTIONNAIRE" (BEBQ), a tool about feeding behavior of infants. UEPG Ci Biol Saúde. 2017;23(2):108-17. http://dx.doi.org/10.5212/Publ. Biologicas.v.23i2.0003.

19. Capurro H, Konichezky S, Fonseca D, Caldeyro-Barcia R. A simplified method for diagnosis of gestational age in the newborn infant. $J$ Pediatr. 1978;93(1):120-2. http://dx.doi. org/10.1016/S0022-3476(78)80621-0. PMid:650322.

20. Associação Brasileira de Empresas de Pesquisa (ABEP). O novo critério padrão de classificação econômica Brasil: critério brasil de classificação econômica. São Paulo: ABEP; 2014.

21. Silveira JA, Colugnati FA, Cocetti $M$, Taddei JA. Secular trends and fators associated with overweight among Brazilian preschool: pnds -1996, and 2006/07. J Pediatr (Rio J); 2014;90:258-66.
22. Wardle J, Guthrie CA, Sanderson S, Rapoport L. Development of the Children's Eating Behaviour Questionnaire. J Child Psychol Psychiatry. 2001;42(7):963-70. http:// dx.doi.org/10.1111/1469-7610.00792. PMid:11693591.

23. The University of British Columbia. [citado 2019 Abr 01]. Disponível em: http://www.stat.ubc.ca/.

24. World Health Organization (WHO). WHO Child Growth Standards: length/ height-for-age, weight-for-age, weightfor-length, weight-for-height and body mass index-for-age: methods and development. Geneva: WHO; 2006. $312 \mathrm{p}$.

25. Villar J, Cheikh IL, Victora CG, Ohuma EO, Bertino E, Altman DG, et al. International standards for newborn weight, length, and head circumference by gestational age and sex: the Newborn Cross-Sectional Study of the INTERGROWTH-21st Project. Lancet. 2014;384(9946):857-68. http://dx.doi. org/10.1016/S0140-6736(14)60932-6. PMid:25209487.

26. Pedreira CE, Pinto FA, Pereira SP, Costa ES. Birth weight patterns by gestational age in Brazil. An Acad Bras Cienc. 2011;83(2):619-25. http://dx.doi.org/10.1590/S000137652011005000008 . PMid:21625798.

27. Smith HA, Becker GE. Early additional food and fl uids for healthy breastfed full-term infants. Cochrane Database Syst Rev. 2016;30(8):CD006462. PMid:27574798.

28. Stettler N, Stallings VA, Troxel $A B$, Zhao J, Schinnar R, Nelson $\mathrm{SE}$, et al. Weight gain in the first week of life and overweight in adulthood: a cohort study of European American subjects fed infant formula. Circulation. 2005;111(15):1897903. http://dx.doi.org/10.1161/01. CIR.0000161797.67671.A7. PMid:15837942.

29. Weng SF, Redsell SA, Swift JA, Yang M, Glazebrook CP. Systematic review and meta-analyses of risk factors for childhood overweight identifiable during infancy. Arch Dis Child. 2012;97(12):1019-26. http://dx.doi. org/10.1136/archdischild-2012-302263. PMid:23109090.

30. Quah PL, Chan YH, Aris IM, Pang WW, Toh JY, Tint MT, et al. Prospective associations of appetitive traits at 3 and 12 months of age with body mass index and weight gain in the 
first 2 years of life. BMC Pediatr. 2015;15(1):153. http://dx.doi. org/10.1186/s12887-015-0467-8. PMid:26459321.
31. Silveira PP, Pokhvisneva I, Gaudreau $\mathrm{H}$, Atkinson L, Flemin AS, Sokolowisk $\mathrm{MB}$, et al. Fetal growth interacts with multilocus genetic score reflecting dopamine signaling capacity to predict spontaneous sugar intake in children. Appetite. 2017;1(120):596-601.

PMid:29038017.

Recebido: $1 \mathrm{abr}, 2019$ Aceito: 4 jun, 2019 